\title{
2D MOF Modulated Fiber Nanogenerator for Effective Acoustoelectric Conversion and Human Motion Detection
}

Krittish Roy ${ }^{\dagger}$, Srikanta Jana ${ }^{\S}$, Zinnia Mallick ${ }^{\perp}$, Sujoy Kumar Ghosh ${ }^{\dagger}$, Basudeb Dutta ${ }^{\S}$, Subrata Sarkar $^{\dagger}$, Chittaranjan Sinha ${ }^{\S, *}$, Dipankar Mandal ${ }^{\perp, *}$

†Department of Physics, Jadavpur University, Kolkata 700032, India

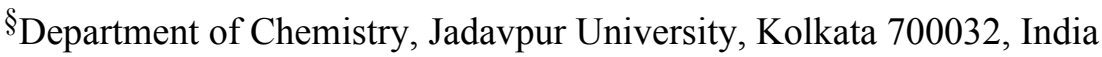

${ }^{\perp}$ Institute of Nano Science and Technology, Knowledge City, Sector 81, Mohali 140306, India

*Corresponding Authors

E-mail: DM:dmandal@inst.ac.in; CRS: crsjuchem@gmail.com 
Table S1. Selected bond lengths and bond angles in $\mathrm{CdI}_{2}-\mathrm{NAP}$ (CCDC-1904947).

\begin{tabular}{|l|l|l|l|}
\hline $\mathrm{I}(1)-\mathrm{Cd}(1)$ & $2.8341(6)$ & $\mathrm{Cd}(1)-\mathrm{I}(1)-\mathrm{Cd}(1) \mathrm{b}$ & $92.89(2)$ \\
\hline $\mathrm{I}(1)-\mathrm{Cd}(1) \mathrm{b}$ & $3.3160(6)$ & $\mathrm{I}(1)-\mathrm{Cd}(1)-\mathrm{N}(1)$ & $98.50(10)$ \\
\hline $\mathrm{Cd}(1)-\mathrm{N}(1)$ & $2.353(4)$ & $\mathrm{I}(1)-\mathrm{Cd}(1)-\mathrm{I}(1) \mathrm{a}$ & $92.89(1)$ \\
\hline $\mathrm{Cd}(1)-\mathrm{N}(1) \mathrm{d}$ & $2.353(4)$ & $\mathrm{I}(1)-\mathrm{Cd}(1)-\mathrm{I}(1) \mathrm{c}$ & $171.48(2)$ \\
\hline $\mathrm{N}(1)-\mathrm{C}(2)$ & $1.438(6)$ & $\mathrm{I}(1)-\mathrm{Cd}(1)-\mathrm{I}(1) \mathrm{d}$ & $95.63(2)$ \\
\hline $\mathrm{C}(1)-\mathrm{C}(2)$ & $1.415(7)$ & $\mathrm{I}(1)-\mathrm{Cd}(1)-\mathrm{N}(1) \mathrm{d}$ & $101.69(11)$ \\
\hline $\mathrm{C}(1)-\mathrm{C}(1) \mathrm{f}$ & $1.434(6)$ & $\mathrm{N}(1)-\mathrm{Cd}(1)-\mathrm{I}(1) \mathrm{a}$ & $76.98(11)$ \\
\hline $\mathrm{C}(1)-\mathrm{C}(5) \mathrm{f}$ & $1.418(6)$ & $\mathrm{N}(1)-\mathrm{Cd}(1)-\mathrm{I}(1) \mathrm{c}$ & $79.72(10)$ \\
\hline $\mathrm{C}(2)-\mathrm{C}(3)$ & $1.365(7)$ & $\mathrm{N}(1)-\mathrm{Cd}(1)-\mathrm{I}(1) \mathrm{d}$ & $101.69(11)$ \\
\hline $\mathrm{C}(3)-\mathrm{C}(4)$ & $1.409(7)$ & $\mathrm{N}(1)-\mathrm{Cd}(1)-\mathrm{N}(1) \mathrm{d}$ & $149.76(14)$ \\
\hline $\mathrm{C}(4)-\mathrm{C}(5)$ & $1.359(8)$ & $\mathrm{I}(1) \mathrm{a}-\mathrm{Cd}(1)-\mathrm{I}(1) \mathrm{c}$ & $78.59(2)$ \\
\hline $\mathrm{I}(1) \mathrm{c}-\mathrm{Cd}(1)-\mathrm{N}(1) \mathrm{d}$ & $76.98(11)$ & $\mathrm{I}(1) \mathrm{a}-\mathrm{Cd}(1)-\mathrm{I}(1) \mathrm{d}$ & $171.48(2)$ \\
\hline $\mathrm{I}(1) \mathrm{d}-\mathrm{Cd}(1)-\mathrm{N}(1) \mathrm{d}$ & $98.50(10)$ & $\mathrm{I}(1) \mathrm{a}-\mathrm{Cd}(1)-\mathrm{N}(1) \mathrm{d}$ & $79.72(10)$ \\
\hline $\mathrm{Cd}(1)-\mathrm{N}(1)-\mathrm{C}(2)$ & $122.3(3)$ & $\mathrm{I}(1) \mathrm{c}-\mathrm{Cd}(1)-\mathrm{I}(1) \mathrm{d}$ & $92.89(1)$ \\
\hline $\mathrm{Cd}(1)-\mathrm{N}(1)-\mathrm{H}(1 \mathrm{~A})$ & 107 & $\mathrm{Cd}(1)-\mathrm{N}(1)-\mathrm{H}(1 \mathrm{~B})$ & 107 \\
\hline
\end{tabular}

$\mathrm{a}=\mathrm{x},-1+\mathrm{y}, \mathrm{z} \quad \mathrm{b}=\mathrm{x}, 1+\mathrm{y}, \mathrm{z} \quad \mathrm{c}=1-\mathrm{x},-1+\mathrm{y}, 3 / 2-\mathrm{z} \quad \mathrm{d}=1-\mathrm{x}, \mathrm{y}, 3 / 2-\mathrm{z} \quad \mathrm{f}=1 / 2-$

$\mathrm{x}, 3 / 2-\mathrm{y}, 1-\mathrm{z}$

Note S1. Preparation of nanofibers.

Firstly, a spinning solution of pure PVDF was prepared by dissolving PVDF pellets in a mixed solvent of 6:4 volume ratio of $\mathrm{N}, \mathrm{N}$-dimethylformamide (DMF)/acetone at a polymer/solvent concentration of $12 \% \mathrm{w} / \mathrm{v}$ under continuous stirring at $60{ }^{\circ} \mathrm{C}$ for $3 \mathrm{~h}$ until a clear homogeneous solution was obtained. Afterwards, as-synthesized MOF (1 wt \% (w/v)) was added into the pure PVDF solution and kept for gentle stirring at $60{ }^{\circ} \mathrm{C}$ prior to complete dissolution. Finally, it was sonicated for some time before electrospinning. Additionally, pure PVDF solution was also taken for electrospinning as a reference. The electrospinnig process was conducted by filling the resulting solution in a $10 \mathrm{~mL}$ hypertonic syringe (Dispovan) tipped with a stainless steel needle $(22 \mathrm{G})$. The positive electric field/bias voltage between tip to $\mathrm{Al}$ foil wrapped grounded collector plate (at a distance of $12 \mathrm{~cm}$ ) was maintained at $10 \mathrm{kV}$ 
with a constant flow rate of $0.6 \mathrm{~mL} / \mathrm{h}$. Moreover, a relative humidity of $40-50 \%$ was adjusted throughout the experiment. Same parameters were maintained during the electrospinning of pure PVDF solution for executing controlled experiments. In addition, collected NPV and composite fibers were further dried at $60{ }^{\circ} \mathrm{C}$ for $4 \mathrm{~h}$ to remove all the residual solvents to obtain stable nanofibers for characterization and device fabrication.

Note S2. Quantification of total electroactive phase content $\left(F_{E A}\right)$ and $\beta, \gamma$ phase content.

The $F_{E A}$ along with relative proportion of $\beta$ and $\gamma$ phase (i.e.; $F(\beta)$ and $F(\gamma)$ ) can be evaluated using the following equations.

$$
\begin{aligned}
F_{E A} & =\frac{A_{841}}{\left(\frac{K_{841}}{K_{764}}\right) A_{764}+A_{E A}} \times 100 \% \\
F(\beta) & =F_{E A} \times\left(\frac{A_{\beta}}{A_{\beta}+A_{\gamma}}\right) \\
F(\gamma) & =F_{E A} \times\left(\frac{A_{\gamma}}{A_{\beta}+A_{\gamma}}\right)
\end{aligned}
$$

where, $A_{841}$ and $A_{764}$ imply the absorbance intensities at $841 \mathrm{~cm}^{-1}$ and $764 \mathrm{~cm}^{-1}$, respectively. $\mathrm{K}_{841}\left(7.7 \times 10^{4} \mathrm{~cm}^{2} \mathrm{~mol}^{-1}\right)$ and $\mathrm{K}_{764}\left(6.1 \times 10^{4} \mathrm{~cm}^{2} \mathrm{~mol}^{-1}\right)$ are the absorption coefficients concerning respective wavenumbers. $A_{\beta}$ and $A_{\gamma}$ indicate the intensities regarding the $\beta$ and $\gamma$ phases under the deconvoluted curves of the $841 \mathrm{~cm}^{-1}$ main peak.

Note S3. Calculation of degree of crystallinity.

Total degree of crystallinity $\left(\chi_{C}\right)$ of each sample is calculated using the following equation.

$$
\chi_{C}=\frac{\sum A_{c r}}{\sum A_{c r}+\sum A_{a m r}}
$$

Where, $\Sigma A_{c r}$ and $\Sigma A_{\text {amr }}$ denote the collective whole integral areas related to the crystalline peaks and the amorphous halo, respectively. 

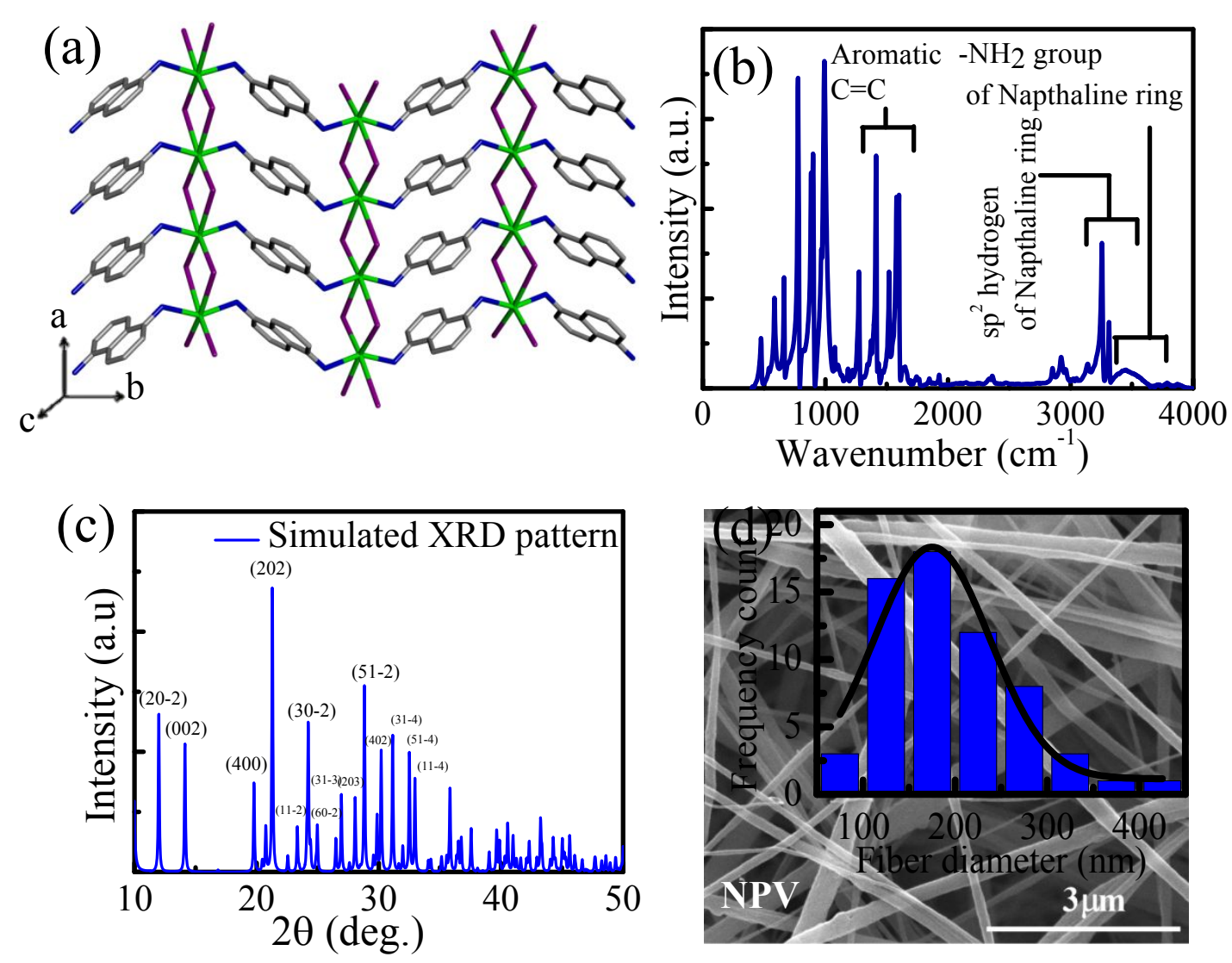

Figure S1. (a) 2D layered structure, (b) FT-IR spectra (in the wavenumber range of 4000-400 $\mathrm{cm}^{-1}$ ) and (c) simulated XRD pattern (extracted from single crystal data) of the as-synthesized $\mathrm{CdI}_{2}$-NAP. (d) FE-SEM image of NPV fiber along with its fiber diameter distribution in the upper inset. 

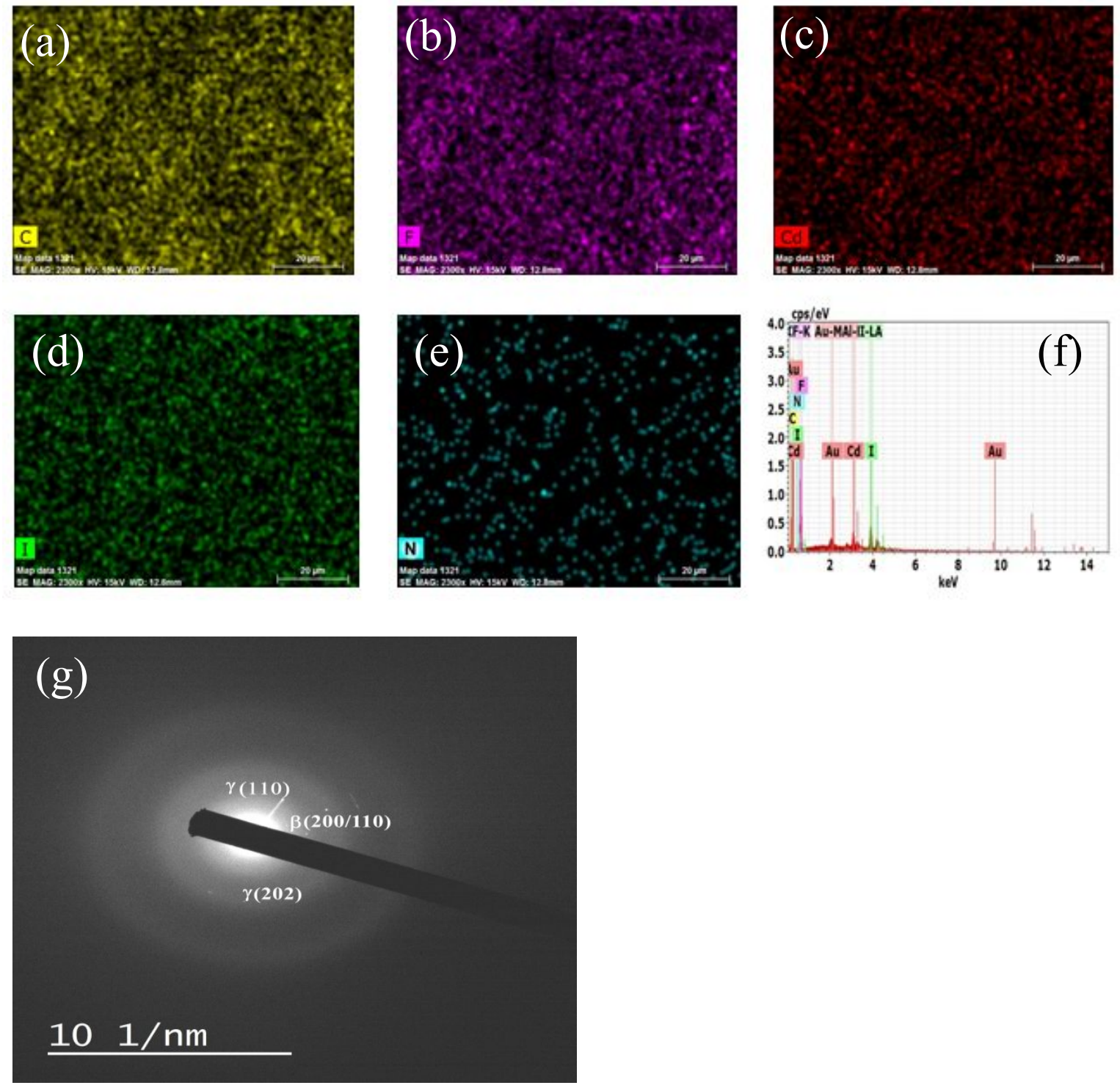

Figure S2. (a) Elemental mapping image of (a-e) carbon (C), fluorine (F), cadmium (Cd), iodine (I), nitrogen (N) in the composite fiber. (f) EDX spectra and (g) SAED pattern of composite fiber. 

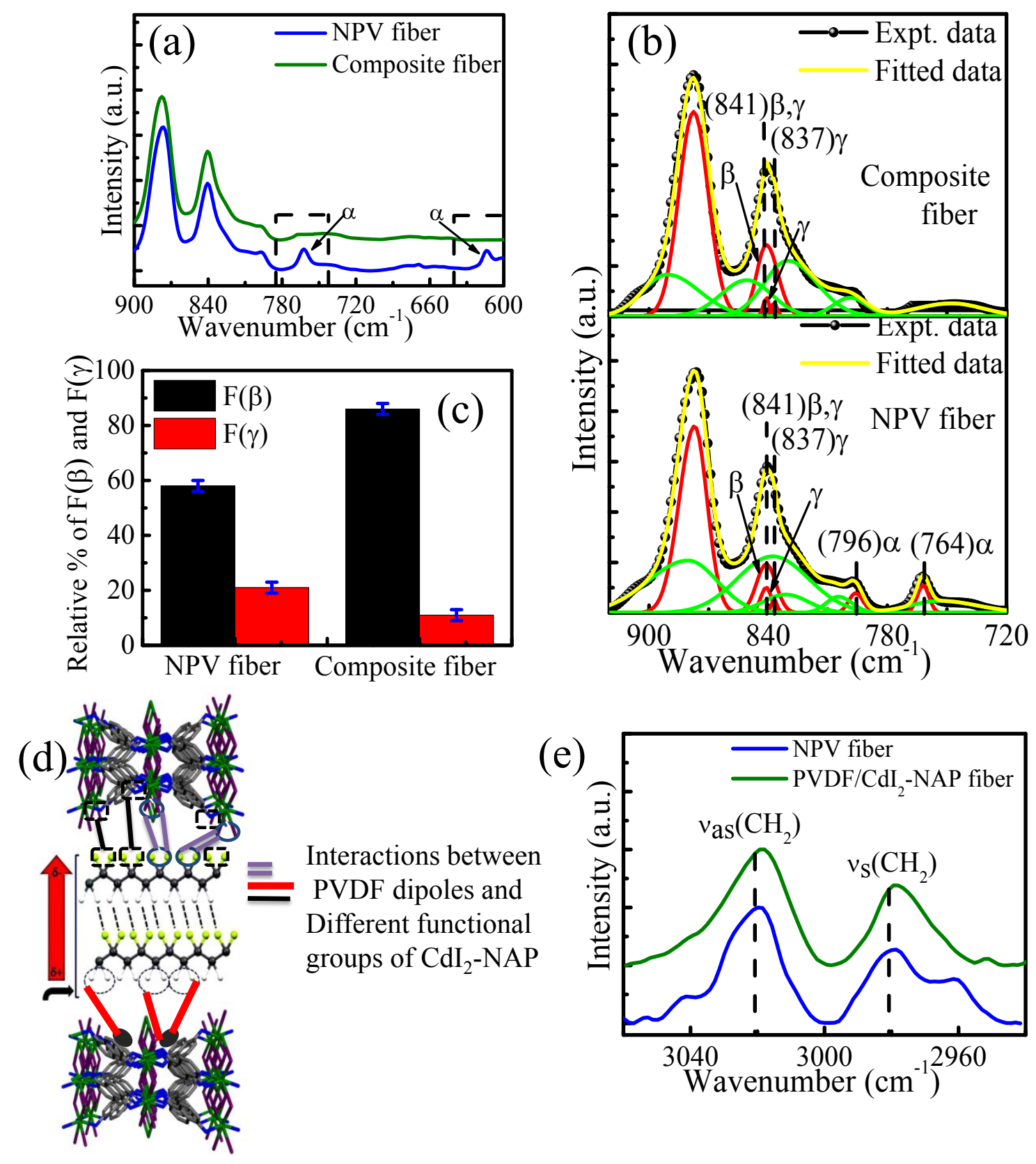

Figure S3. (a) FT-IR spectra in the range of $900-600 \mathrm{~cm}^{-1}$ (b) deconvoluted FT-IR spectra in the range of $920-720 \mathrm{~cm}^{-1}$ (c) relative proportion of $\beta$ and $\gamma$-phase and (d) Schematic demonstration of MOF and PVDF interaction. (e) FT-IR spectra in the range of 3060-2940 $\mathrm{cm}^{-1}$ for composite and NPV fiber. 

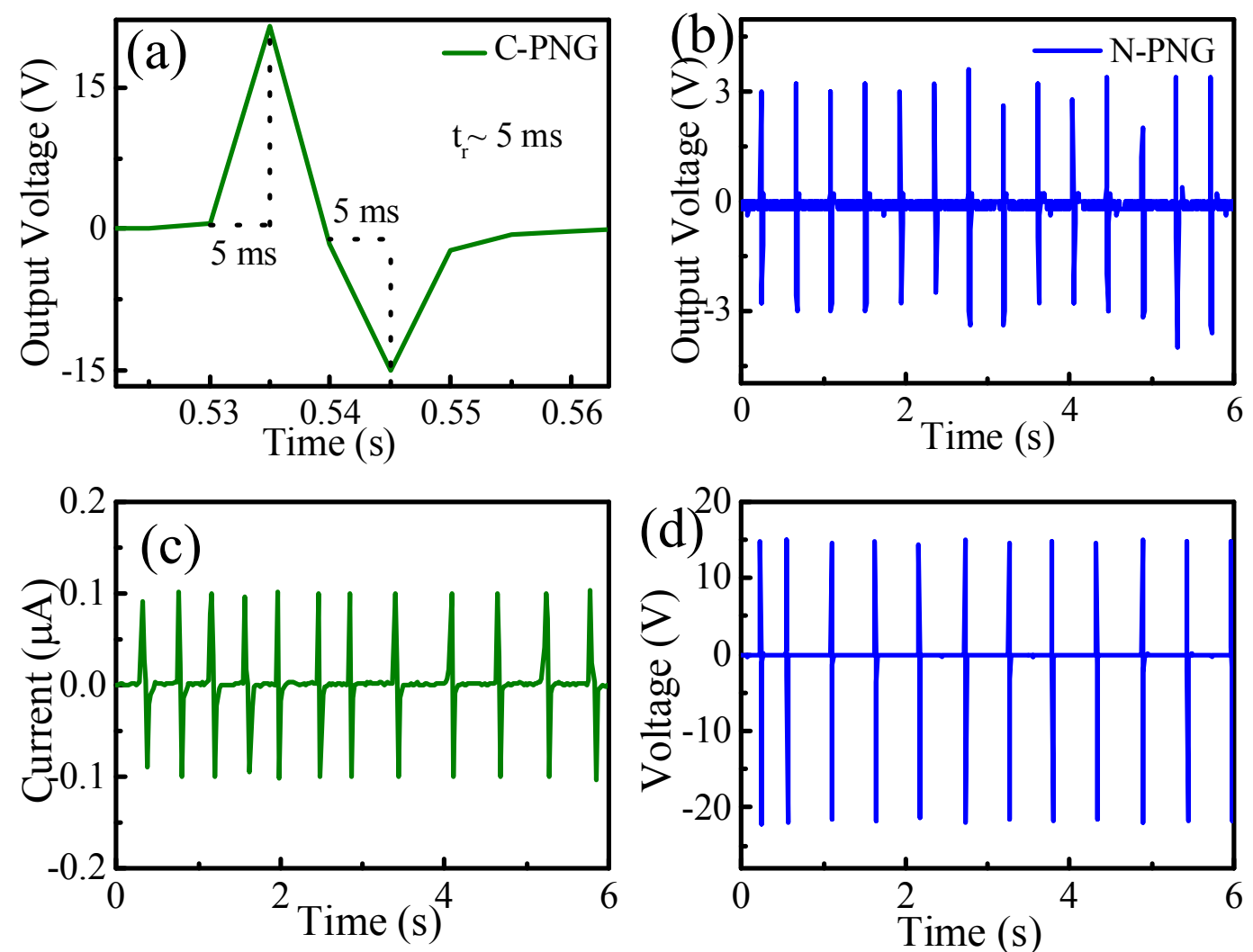

Figure S4. (a) Enlarged view of one single peak of open-circuit voltage generated from CPNG. (b) Open-circuit output voltage $\left(V_{o c}\right)$ from N-PNG during repetitive hand imparting (under compressive stress of $22 \mathrm{kPa}$ ). (c) Short-circuit current $\left(I_{s c}\right)$ under same pressure amplitude. (d) Open-circuit output voltage of C-PNG with reverse electrode polarity. 

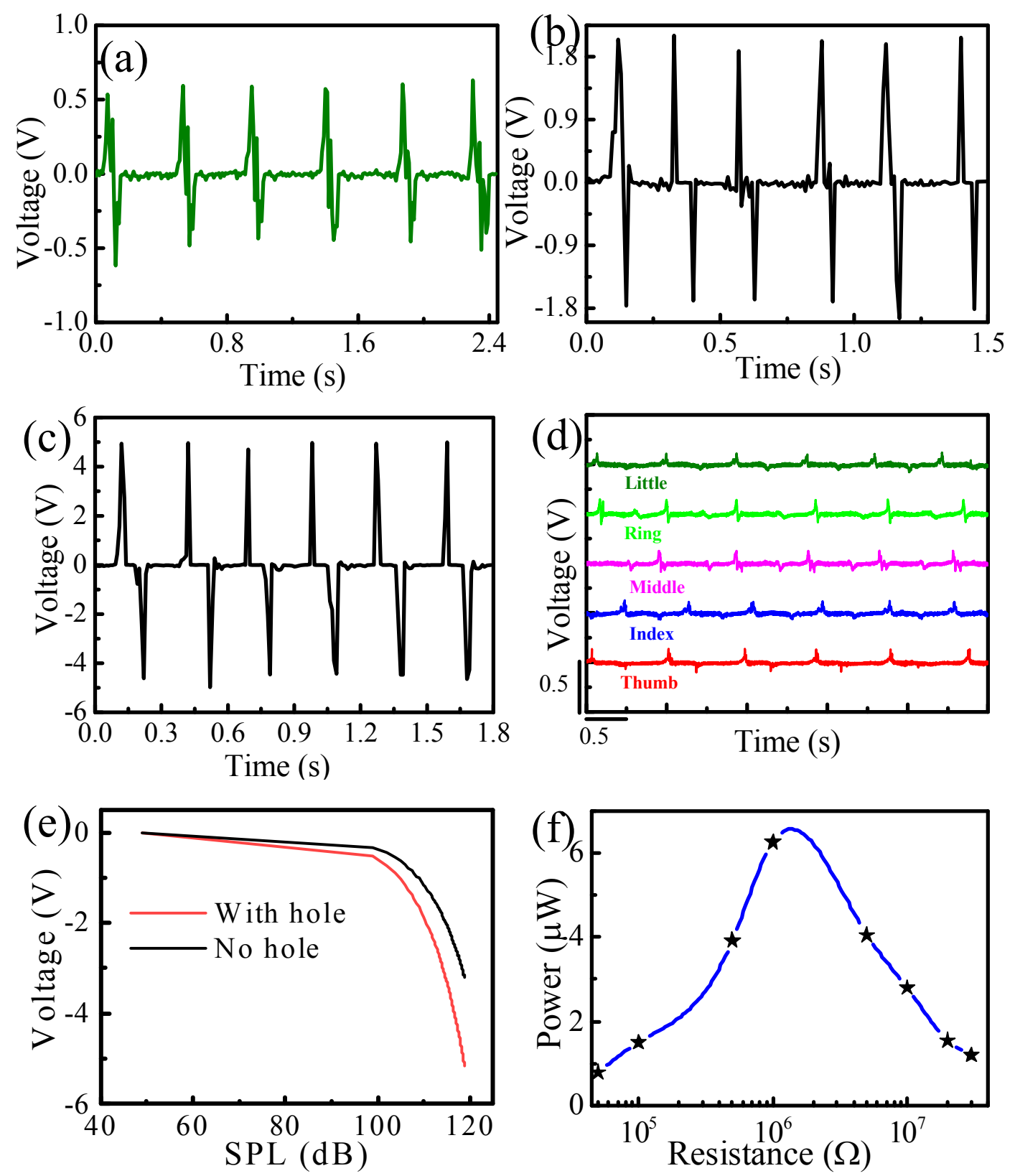

Figure S5. Output voltage signal generated from C-PNG attached under insole of shoe during (a) walking, (b) jogging and (c) jumping. (d) Finger motion monitoring when C-PNG attached to different hand fingers. (e) FEM based theoretical analysis: SPL dependency of acoustoelectric output voltage. (f) Acoustoelectric output power of C-PNG. 